\title{
Muography background sources: simulation, characterization, and machine-learning rejection
}

\author{
J. Peña-Rodríguez, ${ }^{a, *}$ R. de'León-Barrios, ${ }^{a}$ A. Ramírez-Muñóz, ${ }^{b}$ D. Villabona-Ardila, ${ }^{b}$ \\ M. Suárez-Durán, ${ }^{c}$ A. Vásquez-Ramírez, ${ }^{a}$ H. Asorey ${ }^{d}$ and L. A. Núñez ${ }^{a}$ \\ ${ }^{a}$ Universidad Industrial de Santander, Escuela de Física \\ Calle 9 Carrera 27, Bucaramanga, Colombia \\ ${ }^{b}$ Universidad Industrial de Santander, Escuela de Sistemas \\ Calle 9 Carrera 27, Bucaramanga, Colombia \\ ${ }^{c}$ Université Libre de Bruxelles \\ Belgium Boulevard du Triomphe, 21050 Brussels, Belgium \\ E-mail: jesus.pena@correo.uis.edu.co
}

\begin{abstract}
Muography scans large-size objects, natural or anthropic, by recording the atmospheric muon flux crossing them. The traversing muon flux, four orders of magnitude lower than the vertical muon flux, suffers an overwhelming background. The background sources are scattered muons, electromagnetic particles, reverse trajectory particles, and particles arriving simultaneously. We carried out Monte Carlo simulations to characterize such background sources. We estimated the scattered muon energy-angular spectrum and the cosmic ray components impinging the Muon Telescope-MuTe. We quantified the muography background using the Time-of-Flight and Water Cherenkov detector measurements of MuTe. We explored machine learning techniques to reject the background events.
\end{abstract}

$37^{\text {th }}$ International Cosmic Ray Conference (ICRC 2021)

July 12th - 23rd, 2021

Online - Berlin, Germany

\footnotetext{
${ }^{*}$ Presenter
} 


\section{Muography background sources}

Absorption muography employs traversing muon flux for imaging inner density distributions of geological or archaeological structures. However, the low muon fluxes make this technique susceptible to several background sources. This background comes from low-energy muons scattered by the target surface, backward going particles, electromagnetic particles from extensive air showers, and particles impinging simultaneously.

\subsection{Scattered muons}

Muography target structures change the incident trajectory of low energy muons by multiple Coulomb scattering [1-4]. The scattering angle depends on the muon energy, the traversed distance and the material density. The incident muon must have an energy of $\sim 4 \mathrm{GeV}, 60 \mathrm{GeV}$, and $1 \mathrm{TeV}$ to cross $10 \mathrm{~m}, 100 \mathrm{~m}$, and $1000 \mathrm{~m}$ of standard rock $\left(2.6 \mathrm{gcm}^{-3}\right)$ respectively. The angular variation reaches $1^{\circ}(17.4 \mathrm{mrad})-2^{\circ}(35 \mathrm{mrad})$ for muons with energies $\sim 10 \mathrm{GeV}$ crossing $10 \mathrm{~m}$ of standard rock [5].

The angular variation of the crossing muons causes a blurring effect in the muogram, hiding details in the inner structure of the scanned target. An angular deviation of $35 \mathrm{mrad}$ can generate a spatial variation $\sim 35 \mathrm{~m}$ if the muon telescope is one $\mathrm{km}$ away from the target. When the telescope lies near the target base, a shadow effect can emerge in the muogram. This phenomenon occurs due to muons scattered by the hillsides of the geological structure [6]. This effect appears as high transmittance (low density) regions in the muogram. A $35 \mathrm{mrad}$ deviated muon on a $30^{\circ}$ inclined surface impinges at $13 \mathrm{~m}$ from the target base. The muon telescope must lay at $0.1-1 \mathrm{~km}$ from the target to decrease the shadow effect [7].

Another approach is to establish a muon momentum threshold when the telescope observation distance is unchangeable. The momentum threshold depends on the telescope's location. The closer the distance to the base, the larger the threshold. For 6-24 $\mathrm{m}$ a minimum momentum cutoff of $5 \mathrm{GeV}$ is recommended [2] (deviation $\sim 1.5^{\circ}$ ). For a distance of $500 \mathrm{~m}$ the momentum cutoff was found $1 \mathrm{GeV}$ (deviation $\sim 3.5^{\circ}$ ) [8].

\subsection{Inverse flux}

Muon telescopes can be affected by particles entering from the backside. Such particles are mainly muons $(\sim 10 \mathrm{GeV} / \mathrm{c})[1]$ or particles scattered by the earth's surface. The particles trace trajectories like forward-going muons in telescopes without direction measurement systems [9].

The inverse flux increases when the telescope lies in a place where any structure protects the telescope's rear side against particles. Some measurements have shown the inverse flux rises to $50 \%$ for an elevation angle of the telescope of $<15^{\circ}$.

\subsection{Electromagnetic component of extensive air showers}

After muons, the most abundant secondary particles from extensive air showers at ground level are gammas, positrons, and electrons. Gammas are neutral but charged electromagnetic particles (electron/positrons) contribute to muography background [10, 11]. Pair production and singlephoton bremsstrahlung mechanisms create the electromagnetic cascade along the atmosphere. 
These particles have short path lengths but reach long atmospheric depths by consecutive steps of creation/annihilation.

Electromagnetic particles cause muography background in two ways, random coincidences in periods shorter than the detector timing [12] or single electromagnetic particles with enough energy to cross the entire detector mimicking a muon signal.

\subsection{Multiple particle events}

Charged particles from cosmic ray background simultaneously impinge on muon telescopes generating coincidences like single muons traversing the detector. Installation of multiple detection panels reduces such a background source but increases the cost.

Correlated particles (i.e. from the same air shower) have relative arrival times in the order of typical detector timings (tens of ns) $[13,14]$. This feature limits the telescope to recognize between a trace of a single muon and a multiple-particle detection. Muon pairs generally compose correlated particles. They can traverse long distances in the atmosphere after depart from an extensive air shower. The acquisition system rejects charged particles impinging randomly (uncorrelated) on the telescope because of their microsecond-order relative arrival time.

\section{Monte Carlo simulations}

\subsection{Cosmic ray secondary flux}

We carried out Monte Carlo simulations to estimate the spectrum of secondary cosmic rays at ground level. We used the CORSIKA (COsmic Ray SImulations for KAscade) program for detailed simulation of extensive air showers. We chose the Cerro Machin volcano (2650 m a.s.l.), located on the Andean mountain range in the southwest of Colombia, as the observation place.

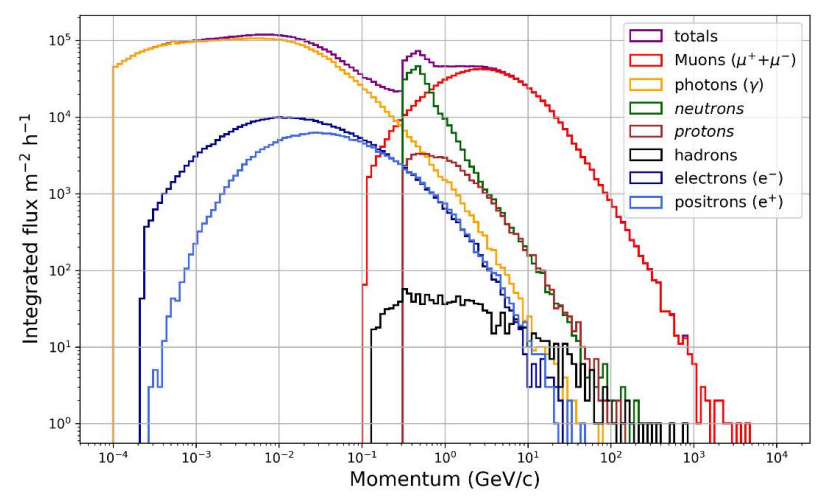

Figure 1: Simulated secondary cosmic ray spectrum at the Cerro Machin volcano [15]. Two humps prevail, one electromagnetic $\left(e^{ \pm}, \gamma\right)$ centered in $\sim 20 \mathrm{MeV} / \mathrm{c}$ and one muonic $\left(\mu^{ \pm}\right)$at $\sim 3 \mathrm{GeV} / \mathrm{c}$.

The spectrum of secondary particles has two principal humps overlapped in $0.3 \mathrm{GeV} / \mathrm{c}$. The electromagnetic component ( $e^{ \pm}$and $\gamma$ ) shapes the first hump with an average momentum $\sim 20 \mathrm{MeV} / \mathrm{c}$. The second hump represents the muonic component $\left(\mu^{ \pm}\right)$. The muon momentum average is $\sim 3 \mathrm{GeV} / \mathrm{c}$. Neutrons peak at $\sim 500 \mathrm{MeV} / \mathrm{c}$ but are undetectable for muon telescopes. Hadron flux 
(pions and kaons) reaches three magnitude orders less than muons being a negligible background source.

The spectrum confirms the electromagnetic particles can influence muography background because of their abundance at ground level. A $10 \mathrm{MeV}$ electron can cross a muon telescope, causing a signal like emerging muons from the scanned target.

\subsection{Muon Forward Scattering}

Muon forward scattering (MFS) contributes to muography background sources. This phenomenon deviates muons after interacting with the structure surface, mimicking trajectories of muons crossing it [2]. We carried out a series of simulations using the Geant4 software. We designed a Geant 4 environment where we created a standard rock horizontal cuboid as the ground surface. In that environment, we simulated the interaction of muons with the ground with different initial conditions: momentum ranging from 0 to $10 \mathrm{GeV} / \mathrm{c}$ with steps of $0.5 \mathrm{GeV} / \mathrm{c}$, and zenith angles from $80^{\circ}$ to $90^{\circ}$ (with respect to the ground's normal) with steps of $0.5^{\circ}$.

We determined the MFS probability (for muons arriving with a given zenith angle and momentum) by counting the number of scattered muons and dividing it by the total number of muons per beam. The atmospheric muon background was simulated according to the methodology in [16], i. e. including corrections by the geomagnetic field.

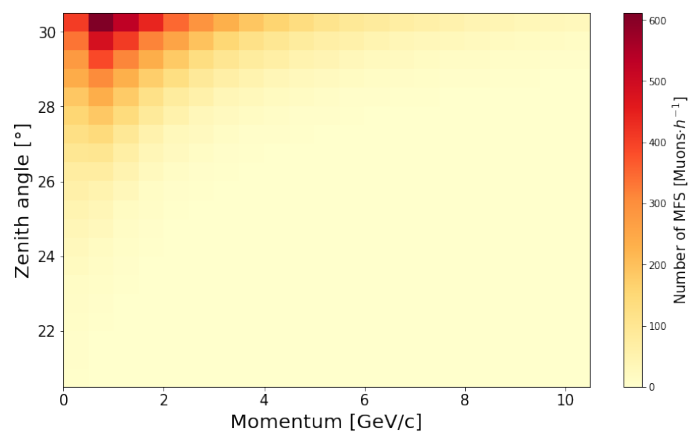

Figure 2: Estimated number of MFS. Here, the muon flux is shown as if it had interacted with a $\sim 59^{\circ}$ slope on the ground, since we estimated that would be were the MFS would be at its maximum. The vertical axis shows the arrival zenith angle of the muons not with respect to this slope, but with respect to a vertical, so the maximum coincides with $30^{\circ}$. We concluded that the effect of MFS is negligible for muon momentum above $\sim 5 \mathrm{GeV} / \mathrm{c}$ and incidence zenith angles smaller than $\sim 85^{\circ}$ with respect to the surface's normal.

From the background of atmospheric muons, we determined that about $88 \%$ of them arrive with momentum below $10 \mathrm{GeV} / \mathrm{c}$. The muon flux peaks at a zenith angle around $30^{\circ}$. Since the occurrence of MFS depends on the zenith angle, it's clear that the probability of MFS for atmospheric muons increases according to the slope of the ground. Our preliminary simulations showed that the MFS effect is greater at an incidence angle of $>99^{\circ}$ with respect to the surface's normal. Taking into account the peak flux of atmospheric muons at $\sim 30^{\circ}$, we estimated that the MFS phenomenon would be maximum if the ground slope was $\sim 59^{\circ}$. A perfectly horizontal ground surface would result in a negligible MFS effect due to the small flux of near-horizontal atmospheric muons. In figure 2 we see the estimated number of MFS by interacting with a $\sim 59^{\circ}$ slope. From figure 2, we see that the MFS phenomenon is only significant for muons arriving with momentum 
smaller than $\sim 5 \mathrm{GeV} / \mathrm{c}$ and zenith angles greater than $\sim 85^{\circ}$, with MFS cases accounting about $22.70 \%$.

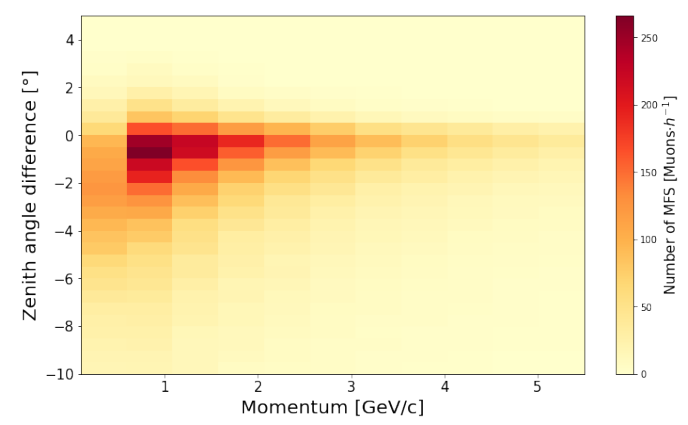

(a) Deflection in zenith angle with respect to momentum.

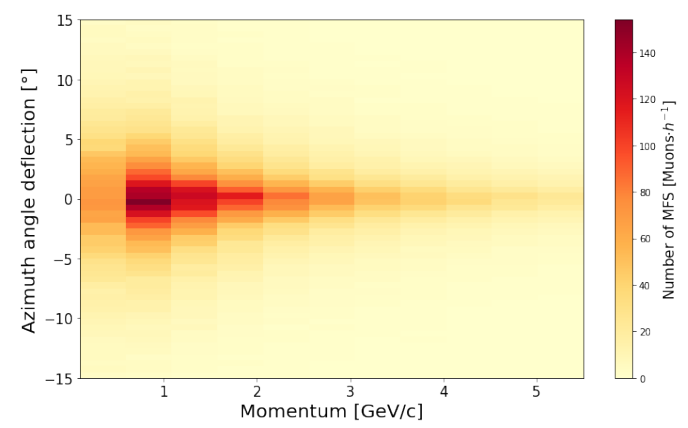

(b) Deflection in azimuth angle with respect to momentum.

Figure 3: Deflection of atmospheric muons in zenith and azimuth angles after MFS. The largest deflections occur for low momentum muons while being less common for muons with higher momentum. These results are consistent with findings shown in [2]

Figure 3 shows the deflection in zenith $(\Delta \theta)$ and azimuth $(\Delta \phi)$ angles, for scattered muons with respect to their momentum. This deflection is defined as the final zenith/azimuth angle minus the initial one. The figure shows muons with initial momentum below $\sim 5.5 \mathrm{GeV} / \mathrm{c}$ and incidence zenith angles above $85^{\circ}$. In both figures, the largest deflections occur for muons with lower energies. Still, the majority of muons undergo small deflections such that $69.52 \%$ of MFS cases have $-5^{\circ} \leq \Delta \theta \leq 5^{\circ}$ and $67.16 \%$ of MFS cases have $-5^{\circ} \leq \Delta \phi \leq 5$. The results obtained in this section of our work are consistent and complementary with those shown in [2].

\section{Background measurements}

\subsection{Electromagnetic and multiple-particle background}

The MuTe distinguishes between electromagnetic particles and muons by using measurements of deposited energy from the water Cherenkov detector (WCD) installed behind the scintillator hodoscope [17]. Figure 4 (left) shows the deposited energy spectrum of particles detected in coincidence Hodoscope-WCD. We divided the spectrum into three components, electromagnetic, muonic, and multiple particle. The deposited energy of electromagnetic particles is below $180 \mathrm{MeV}$. The muonic hump ranges $180 \mathrm{MeV}<E_{d}<400 \mathrm{MeV}$ with an average deposited energy of $240 \mathrm{MeV}$ (Vertical Muon Equivalent - VEM).

Events in the multiple particle hump deposit energies above $400 \mathrm{MeV}$-deposited energy of a corner-to-corner crossing muon. Most of such events deposit $\sim 480 \mathrm{MeV}$ which is equivalent to the signal of two crossing muons. These particles arrive at the muon telescope with relative times lower than the WCD recording window ( $300 \mathrm{~ns}$ ), indicating their origin in the same EAS.

The Time-of-Flight of particles impinging on MuTe is shown in figure 4 (right). Single and correlated particles have a ToF $<100 \mathrm{~ns}$. No-correlated particles have a relative time $>300 \mathrm{~ns}$. The probability that a non-correlated event occurs below $200 \mathrm{~ns}$ is $\sim 0.05 \%$. 

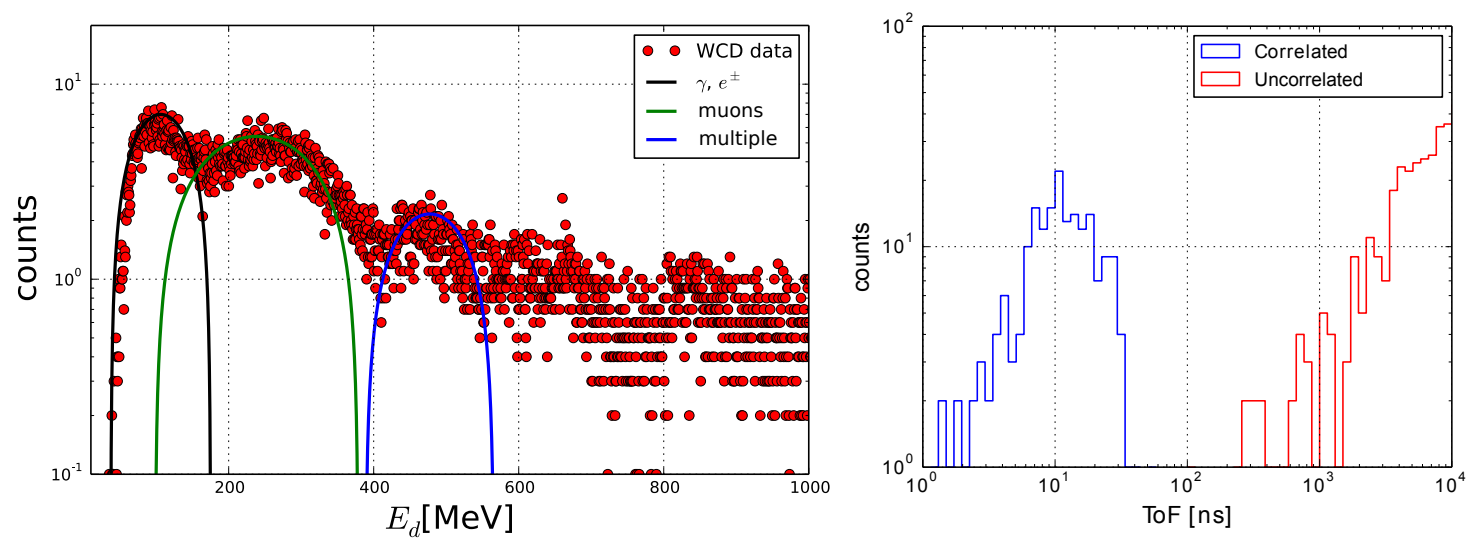

Figure 4: Deposited energy spectrum (left) and Time-of-Flight (right) of particles crossing the MuTe. We observe three kinds of events, electromagnetic (black-line), muonic (green-line), and multiple-particle (blueline). Uncorrelated particles have relative times above $300 \mathrm{~ns}$ while correlated or single-particle events below $100 \mathrm{~ns}$.

\subsection{Inverse flux}

We analyzed a 10-day data recording to evaluate the influence of particles impacting the telescope from the rear side. Figure 5 shows the angular distribution of frontal flux (left) and inverse flux (right). The inverse flux represents $\sim 22 \%$ of the data for an elevation angle of $15^{\circ}$ [18].
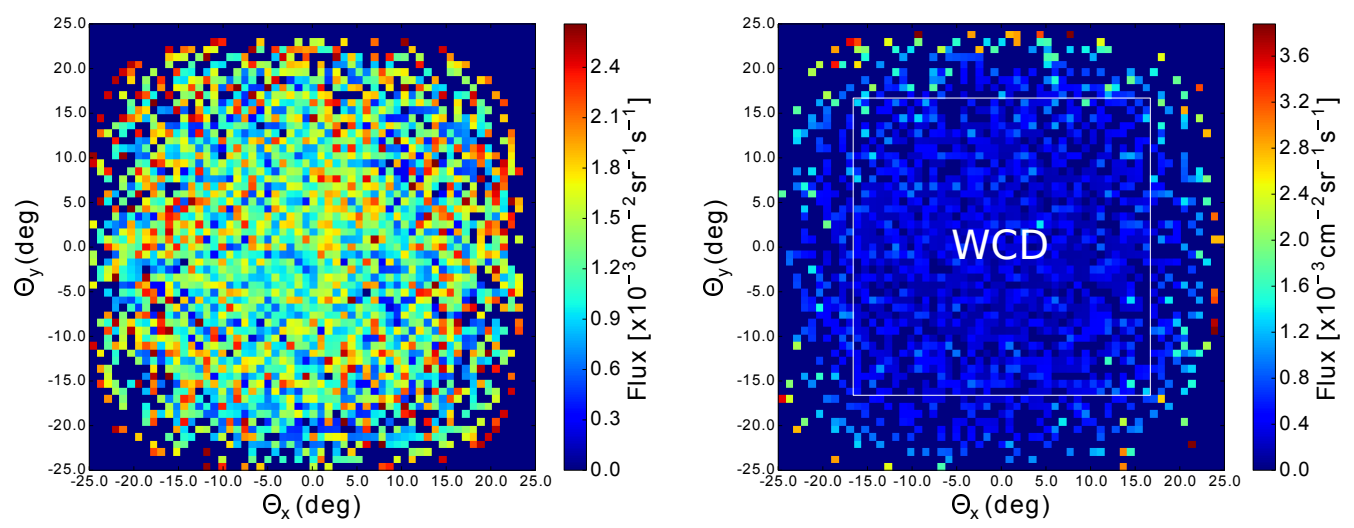

Figure 5: Frontal (left) and inverse (right) particle flux measured by MuTe. The WCD (white-square) filter low energy particles $(<240 \mathrm{MeV})$ entering for the rear side of the MuTe.

The WCD located behind the hodoscope absorbs inverse fluxes of particles with energies below $240 \mathrm{MeV}$. This active absorbent decreases the inverse flux background of the muogram measured by the hodoscope.

The white square indicates the acceptance window between the hodoscope and the WCD. Particles crossing the hodoscope but not the WCD generates a border effect in the flux histogram reaching $\sim 3.87 \times 10^{-3} \mathrm{~cm}^{-2} \mathrm{sr}^{-1} \mathrm{~s}^{-1}$. 


\section{Background rejection with machine learning}

Muon telescopes reduce background by installing absorbent panels (to filter low-energy particles) and increasing the sensitive panel number (to reduce the probability of detecting multipleparticle events). We considered the elimination of muography background combining particle identification and machine learning.

We used a Gaussian Mixture Model (GMM) to parametrize the components of the deposited energy spectrum of particles crossing the WCD. We set 3 background components (electromagnetic, muonic, and multiple-particle) with an initial weight of $0.6,0.3$, and 0.1 respectively. The GMM returns the parameters mean and variance for each component. We applied a spectrum cutoff in $580 \mathrm{MeV}[19]$.

We chose a supervised classification testing Naïve Gaussian, Support Vector Machines (Radial Basis Function), and Random Forest algorithms. The best algorithm (Naïve Gaussian) classifies the electromagnetic (99\%), muonic (100\%), and multiple particle $(97 \%)$ events.

\section{Conclusions}

We found the spectrum of particles impinging on MuTe is mainly composed of muons $(\sim 3$ $\mathrm{GeV} / \mathrm{c}$ average) and electromagnetic particles (electrons, positrons, and gammas with $\sim 20 \mathrm{MeV} / \mathrm{c}$ average). The electromagnetic background contains up to $36 \%$ of the data recorded. The scattering probability of muons increases inversely with the energy and relative incidence angle with respect to the object surface. For muons with momentum $<1 \mathrm{GeV} / \mathrm{c}$, the scattering angle is above 1 degree. Backside entering particles represent up to $22 \%$ of the flux and depend on its elevation angle. Two processes cause the multiple particle background. Independent particles from the atmospheric radiation background with relative arriving times $>300 \mathrm{~ns}$ and correlated particles (mainly a muon pair) originated in the same EAS with relative arriving times $<100 \mathrm{~ns}$.

We found that about $22.70 \%$ of atmospheric muons below $5 \mathrm{GeV} / \mathrm{c}$ and with an incidence zenith angle greater than $85^{\circ}$ with respect to the solid surface will undergo MFS. $67.16 \%$ of these muons will undergo deflections smaller than $5^{\circ}$ for their azimuth angle, while the difference between the final zenith angle after "bouncing" and the initial one will be less than $5^{\circ}$ for $69.52 \%$ of them.

\section{References}

[1] R. Nishiyama et al., Experimental study of source of background noise in muon radiography using emulsion film detectors, Geosci. Instrum. Methods Data Syst. 3 (2014) 29.

[2] H. Gómez et al., Forward scattering effects on muon imaging, J. Instrum. 12 (2017) P12018.

[3] M. Furlan et al., Application of muon tomography to detect radioactive sources hidden in scrap metal containers, in 2013 3rd ANIMMA International Conference, IEEE, jun, 2013, DOI.

[4] S. Pesente et al., First results on material identification and imaging with a large-volume muon tomography prototype, Nucl Instrum Methods Phys Res A. 604 (2009) 738. 
[5] M. Suárez-Durán, Variaciones del flujo de radiación cósmica en distintos escenarios geofísicos, Ph.D. thesis, Universidad Industrial de Santander, 2019.

[6] C. Cârloganu et al., Towards a muon radiography of the puy de dôme, Geosci. Instrum. Methods Data Syst. 2 (2013) 55.

[7] L. Oláh et al., Investigation of the limits of high-definition muography for observation of mt sakurajima, Philos. Trans. A Math. Phys. Eng. Sci. 377 (2018) 20180135.

[8] R. Nishiyama et al., Monte carlo simulation for background study of geophysical inspection with cosmic-ray muons, Geophys. J. Int. 206 (2016) 1039.

[9] K. Jourde et al., Experimental detection of upward going cosmic particles and consequences for correction of density radiography of volcanoes, Geophys. Res. Lett. 40 (2013) 6334.

[10] L. Oláh et al., Investigation of background sources of muography, in PoS(ICRC2017), Sissa Medialab, Aug., 2017, DOI.

[11] L. Oláh et al., Investigation of soft component in cosmic ray detection, Astroparticle Physics 93 (2017) 17.

[12] T. Kusagaya et al., Muographic imaging with a multi-layered telescope and its application to the study of the subsurface structure of a volcano, Proceedings of the Japan Academy, Series B 91 (2015) 501.

[13] L. Bonechi et al., Atmospheric muons as an imaging tool, Reviews in Physics 5 (2020) 100038 .

[14] D. Gámez, Muon arrival time distributions and its relationschip to the mass composition of ultra high energy cosmic rays : an application to the Pierre Auger Observatory, Editorial de la Universidad de Granada, Granada (2011).

[15] A. Vasquez-Ramírez, Estimación de la respuesta generada por el detector mute al paso de partículas cargadas, master's thesis, Universidad Industrial de Santander, 2018.

[16] H. Asorey et al., Preliminary results from the latin american giant observatory space weather simulation chain, Space Weather 16 (2018) 461.

[17] J. Peña-Rodríguez et al., Design and construction of MuTe: a hybrid muon telescope to study colombian volcanoes, Journal of Instrumentation 15 (2020) P09006.

[18] J. Peña-Rodríguez et al., Characterization of the muography background using the muon telescope (MuTe), in PoS(ICHEP2020), Sissa Medialab, Feb., 2021, DOI.

[19] A. Ramírez-Muñoz and D. Villabona-Ardila, Discriminación del ruido de fondo en muografía usando técnicas de aprendizaje automático, bachelor's thesis, Universidad Industrial de Santander, 2021. 